\title{
Imaging of occlusal dental decay With 780 nm NIR light
}

\author{
Faten E. Ali ${ }^{1 *}$ and Ziad T. Al-dahan ${ }^{2}$ \\ Assistant Lecturer, Department of Engineering College, Al-Nahrain University, Baghdad, Iraq ${ }^{1}$ \\ Professor, Department of Engineering College, Al-Nahrain University, Baghdad, Iraq ${ }^{2}$
}

Received: 17-April-2019; Revised: 20-June-2019; Accepted: 25-June-2019

(C)2019 Faten E. Ali and Ziad T. Al-dahan. This is an open access article distributed under the Creative Commons Attribution (CC BY) License, which permits unrestricted use, distribution, and reproduction in any medium, provided the original work is properly cited.

\begin{abstract}
The traditional decay detection methods such as radiographs often are unsuccessful to detect initial enamel lesions that have not progressed to cavitation. Also, radiographs may refuse by patients for safety concerns. Thus, a new technique as intraoral visual camera developed to be useful tools for clinical dentistry, research, and caries detection. This paper aims to evaluate a diagnostic advantage of a new tool in integration with a prototype camera for detection of tooth caries in life images by using near infrared (780 NM) laser source. The results show the translucency of teeth in the near infrared range that offers a non-ionizing and safety to detect dental caries. Our system was tested with numbers of volunteers and successfully detected occluded and early caries lesions without supplementary image processing. Also, the near infrared system can provide details on the precise site of the lesion and also showing the extent of the lesion in enamel but cannot just so determine the lesion depth in dentin. The clinical near infrared system utility, simple design, low cost, and user friendliness make it an active dental caries diagnosis technology, which could aid provide individualized precautionary measures and noninvasively administration of caries and minimize the exposure to unnecessary non-ionizing radiation.
\end{abstract}

\section{Keywords}

Dental (caries/decay), NIR, Laser, Early diagnosis, Intraoral camera.

\section{Introduction}

Tooth decay is an infectious disease process that causes damage to the structure of teeth, is one of the most prevalent clinical conditions in the world. Cavities are decayed areas in the teeth, which result of the metabolism process of bacteria that progressively dissolves a tooth's enamel (hard outer surface) and proceed toward the interior layers. Cavities are the most prominent sequel of dental caries, when left without treatment that leads to pain, loss of a tooth or in very rare cases, death [1]. In spite of markedly changed by the nature of dental caries consequent to the using the fluoride in the water to drink, dentifrices and other applications of improved dental health, dental decay remains the main cause of tooth loss. Tooth caries caused by the oral bacteria metabolize sugars and starches found in food that produce acids that corrode the tooth enamel [2]. Caries are associated with the existence of dental plaque that created by the same antagonizing bacteria [3].

*Author for correspondence

175
Gradually, the layer of enamel is compromised and the acids offense the inner dentin and ultimately the soft pulp [4], causing pain, halitosis, allergy, and stains. Tooth decay can also cause inflammation and infection of the surrounding gingiva. Caries treatment usually done by manually removing the defected tooth material and filling the residual void. The problem of caries nature changed significantly with the plurality of recently known carious lesions which localized on the occlusal surfaces of the proximal contact areas and posterior teeth [5]. Predominating these early decays are hidden in the convoluted topography of the pits and fissures or by debris that much stacks in those regions of the posterior teeth. Furthermore, like this lesion are difficult to detect in the early stages of evolution (Figure 1).

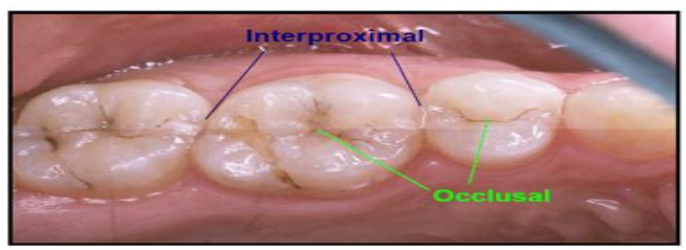

Figure 1 Caries lesions found on interproximal and occlusal surfaces of the teeth 
Radiographs coupling with white visual light examination frequently used to aid in locate and determine the depth of the caries. The most popular radiographic views are bitewing, periapical, and occlusal views. In the traditional 2D imaging radiographs, there is an attenuation of finer detail due to the superimposed material between the film and source that directly affects its radiographic visibility that causing not appearing of early caries and cracks on the X-rays. Moreover, radiographs may make concern amongst patients who endeavor to minimize their exposure to radiation. Patients may refuse radiographs in spite of dentist instructions which causing lack on the valuable diagnostic information.

Over the century there has been a potential to improve visual methods for the diagnosis of proximal and occlusal decay of teeth such as: fiber-optic transillumination (FOTI), intraoral camera, fluorescencebased methods, and optical coherence tomography. Optical trans-illumination has significant interest in dentistry diagnosis, during visual trans-illumination, a carious lesion looks to be a dark consequence of light high scattering and absorption of it. However, sound dental enamel, causing strong scattering of light visible wavelengths rang $(400-700 \mathrm{~nm})$, that prevent imaging through the tooth layers [6]. Near infrared illumination (NIR) is the zone of the electromagnetic spectrum between (0.7-2.0) $\mu \mathrm{m}$. Near infrared light has a smaller scattering coefficient in the normal enamel of teeth than visible light, thus it can penetrate much further on the tooth enamel without scattering [6]. Depending on the wavelength, most NIR light can be transmitted across healthy enamel with marginal scattering; for visible range $(400-700 \mathrm{~nm})$, the absorption of enamel is very low [7]. While for dentin the absorption coefficient is basically independent wavelength with a value of $\left(\mu_{\mathrm{a}}\right.$ $=4 \mathrm{~cm}^{-1}$ ) [8]. In enamel scattering is high in the visible wavelength range of $632 \mathrm{~nm}$ with scattering coefficients $\left(\mu_{\mathrm{s}}\right)$ equal $60 \mathrm{~cm}^{-1}$ reduce with higher wavelength to a value of $\left(2-3 \mathrm{~cm}^{-1}\right)$ at $1310 \mathrm{~nm}$ wavelength [9]. Therefore, enamel is almost transparent in the NIR light with visual attenuation lower than in the visible range. NIR light at $1310 \mathrm{~nm}$ wavelength light appear an optimal for teeth imaging as it makes a balance between water attenuation and enamel. [10]. However, dental caries and demineralization scatter transiting near infrared light and look as dark regions [11]. The depth of caries and other features can be further interrogated when comparing the translucent and dark areas at various angles of view, illumination modes, and NIR wavelengths [12]; Figure 2 illustrates common viewing and illumination methods for NIR dental imaging. Cracks in the tooth are easily visualized in the NIR range as well [13].
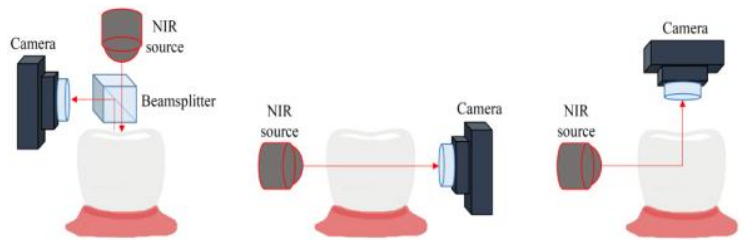

Figure 2 Common viewing and illumination methods for NIR dental imaging

\section{Materials and methods}

\subsection{Device construction}

An intraoral custom 3D printed housing prototype design which is inserted into the mouth has a section of about $15 \mathrm{~mm}$ and a length of $150 \mathrm{~mm}$ and can be rotated 360 degree to simple access the upper and lower teeth. The custom consists of a camera sensitive to near infrared light attached with 90degree mirror to enable horizontal field of view and diode laser of $5 \mathrm{~mW}$ maximum power at a $780 \mathrm{~nm}$ wavelength positioned in 22 degree that direct the light to the teeth surface. The camera uses a CCD sensor with $(1600 \times 1200)$ pixels and can adjust both lighting intensity and focusing rang manually with a 2.0 USB interface which qualify photography and capturing images in JPG format. Speckle noise was minimized by adjusted the power of the laser illumination in the saturation region of contrast that give better near infrared images contrast. The laser source attached to a power supply output cable connected to computer. The costume placed parallel to the teeth surface that allow trans-illumination of the occlusal surface. NIR occlusal trans-illumination produces diagnostic images to NIR imaging systems through its diffuses interior and out the occlusal tooth surface that capable of detecting both occlusal and proximal lesions. Figure 3 shows a diagram of the intraoral custom system.

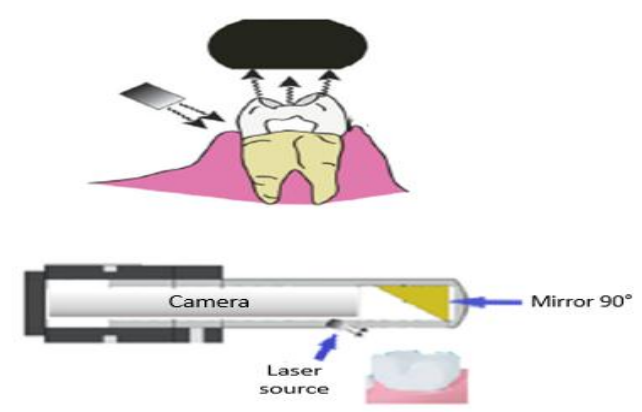

Figure 3 Structure of device construction 


\subsection{Clinical research}

After obtaining a written informed consent, numbers of volunteers were participant aged 12-60 from the patient population. A total of 30 teeth were imaged in the study. Overhead lights were turned off while a dentist imaged sites of interest with both the NIR system and a white light intraoral camera. The NIR system was used to capture approximal and occlusal views in both reflectance and trans-illumination modes. During monitoring a live view of the captured images using the computer, the imaging was utilized in the following configuration:

(i) The intraoral custom used to take conventional images of the teeth using the white visible light.

(ii) The intraoral custom used to take NIR images of the teeth using the NIR laser light for detection of proximal lesions, deeper occlusal lesions and any other outer surface signs of cracks or decay.

During image capturing, the hand piece was moved to get images at several angles which is significant for NIR imaging to avert the interference of specular reflection by the tooth which be visible at normal view and fade at other angles however lesions keep visible for all angles.

\section{Results}

Several extracted teeth with suspected caries were examined using NIR trans-illumination. Figures (4-8) shows images of teeth taken by visible light and NIR, as shows in Figure 4 NIR can provides details on the precise site of the lesion and recognize it from the dental calcification.

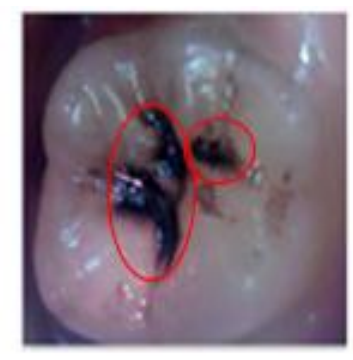

(a)

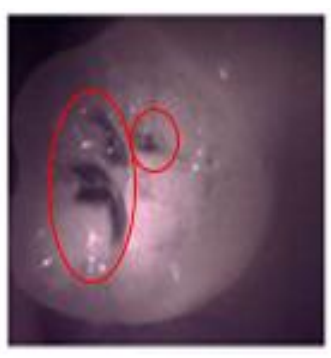

(b)
Figure 4 Tooth images viewing precise areas of lesion. (a) Visible light (b) NIR

Figure 5 illustrate the lesion extension in enamel and how much extents to the dentin, but cannot just so determine the lesion depth in dentin.

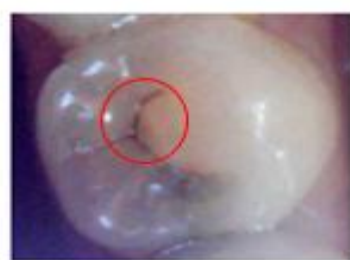

(a)

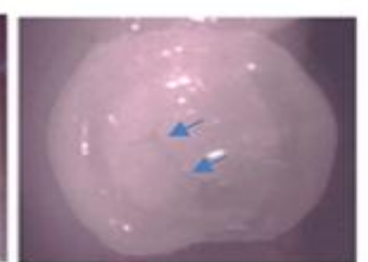

(b)
Figure 5 Image of tooth extent of the lesion in enamel. (a) Visible light (b) NIR

Figure 6 shows clearly location of the lesion and recognizes the decay from the dental calcification.

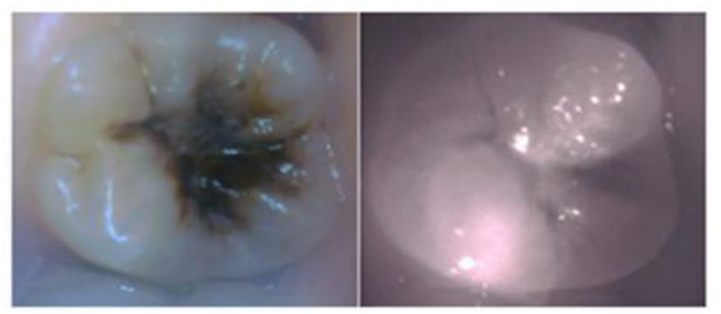

(a)

(b)

Figure 6 Image of tooth shows the location of lesion (a) Visible light (b) NIR

Figure (7 \& 8) shows clearly location of initial cracks that may cause pain or tend to irritate the gums.

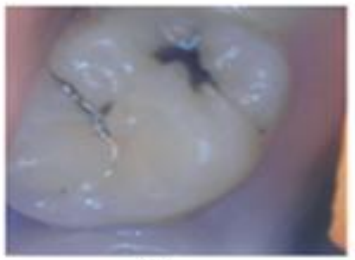

(a)

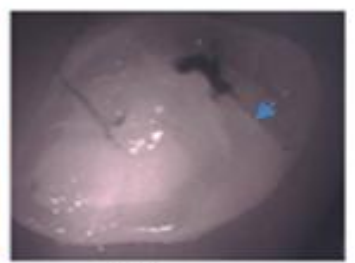

(b)
Figure 7 Image of tooth shows the initial cracks (a) Visible light (b) NIR
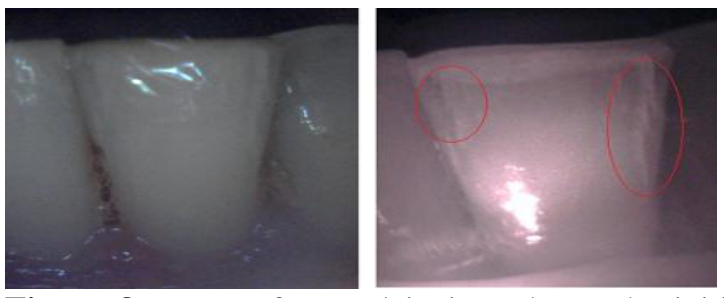

Figure 8 Image of central incisor shows the initial cracks (a) Visible light (b) NIR

\section{Conclusion}

In a study of numbers of volunteers with varying dental conditions, we test a custom NIR system capable of illuminating at $780 \mathrm{~nm}$ and imaging with a digital USB camera. The NIR system was capable of displaying dental decay images that not viewed by 
means of traditional methods such as visual examination and without utilizing ionizing radiation. The superb detail of the images, primarily in comparison to conventional radiographs, potentially grants the clinician greater diagnostic power.

\section{Discussion and future work}

The inexpensive cost of the components required to produce such high-quality images adds a new dimension that is in contrast to conventional imaging approaches. From the results evince near infrared images can utilized for monitoring and detection of occlusal caries lesions where most epochal decay increase, this due to the high transparency of dental enamel to the near infrared light unlike the dentine which has high scattering to the NIR light, this causing a regular distribution of near infrared light diffused under the crowns transparent enamel that produce elevated contrast near infrared images of the occlusal decay and expose unobserved lesions below the surface. Also, usually occlusal surfaces of posterior teeth have stains in the pits and fissures of the its surface which in visible examination not easy to recognize the decay from the dental calcification due to the highly stained surface. While the stain is not visible when using NIR light which enable determine the exact area of decay. In general, NIR laser diode trans-illumination is a reliable and valid method in detecting early and invisible enamel caries and can replace the need for radiography, also could be a powerful diagnostic measure used to allow remineralization of teeth rather than restoring them. Regular diagnosis with NIR could aid provide individualized precautionary measures and noninvasively administration of caries and minimize the exposure to unnecessary non-ionizing radiation.

\section{Acknowledgment}

I would like to thank my supervisor Professor Dr. Ziad Tarik AL-Dahan for his help, advice and support to complete this work. I thank my department Biomedical Engineering in Al Nahrain University for all the support and guidance provided throughout my education.

\section{Conflicts of interest}

The authors have no conflicts of interest to declare.

\section{References}

[1] Subka S, Rodd H, Nugent Z, Deery C. In vivo validity of proximal caries detection in primary teeth, with histological validation. International Journal of Paediatric Dentistry. 2019; 29(4):429-38.

[2] Kianoush N, Adler CJ, Nguyen KA, Browne GV, Simonian M, Hunter N. Bacterial profile of dentine caries and the impact of $\mathrm{pH}$ on bacterial population diversity. PloS one. 2014; 9(3):1-10.

[3] Simón-Soro A, Mira A. Solving the etiology of dental caries. Trends in Microbiology. 2015; 23(2):76-82.

[4] Lenhard M. Restoration of severely compromised teeth with modern operative techniques. Operative Dentistry. 2016; 41(S7):S88-95.

[5] Jones GC, Jones RS, Fried D. Transillumination of interproximal caries lesions with 830-nm light. In lasers in dentistry X 2004 (pp. 17-22). International Society for Optics and Photonics.

[6] Fried D, Staninec M, Darling CL. Near-infrared imaging of dental decay at $1310 \mathrm{~nm}$. Journal of Laser Dentistry. 2010; 18(1):8-16.

[7] Spitzer D, Ten Bosch JJ. The absorption and scattering of light in bovine and human dental enamel. Calcified Tissue Research. 1975; 17(2):129-37.

[8] Ten Bosch JJ, Zijp JR. Dentine and dentine reactions in the oral cavity. Thylstrup A, Leach SA, Qvist V. (IRL Press Ltd, Oxford, England, 1987). 1987:59-65.

[9] Jones RS, Fried D. Attenuation of 1310-and 1550-nm laser light through sound dental enamel. In lasers in dentistry VIII 2002 (pp. 187-90). International Society for Optics and Photonics.

[10] Bühler CM, Ngaotheppitak P, Fried D. Imaging of occlusal dental caries (decay) with near-IR light at 1310-nm. Optics Express. 2005; 13(2):573-82.

[11] Darling CL, Huynh G, Fried D. Light scattering properties of natural and artificially demineralized dental enamel at $1310 \mathrm{~nm}$. Journal of Biomedical Optics. 2006; 11(3).

[12] Fried WA, Fried D, Chan KH, Darling CL. Imaging early demineralization on tooth occlusional surfaces with a high definition in GaAs camera. In lasers in dentistry XIX 2013. International Society for Optics and Photonics.

[13] Fried WA, Simon JC, Lucas S, Chan KH, Darling CL, Staninec M, et al. Near-IR imaging of cracks in teeth. In lasers in dentistry XX 2014. International Society for Optics and Photonics.

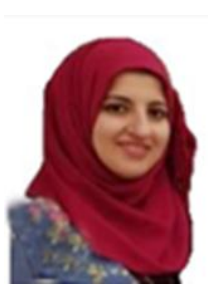

MS. Faten Emad Ali has B.Sc in Biomedical Engineering, College of Engineering, Al-Nahrain University. She is Assistant lecturer at Al-Nahrain University, College of Engineering, Biomedical Engineering Department. She has published scientific \& technical papers, including American Journal of Biomedical Sciences. She has 8 years of academic \& practical's and consulting experience in biomedical researchers. She currently teaches \& conducts research programs in the areas of Medical Equipments, Biomedical Signals, Medical Imaging, Three Dimensional Laser Imaging, Optics Electrical Engineering, Physics Laser and Wireless Sensor Networks.

Email: fatenemadali@gmail.com 
Dr. Ziad T. Al-dahan is a Professor in the Department of Biomedical Engineering at Al-Nahrain University, Baghdad, Iraq. Received the P.G. Diploma (1985), M.Phil. (1986) and Ph.D. (1988) from Brunel University, UK. His research areas are Material Characterization, Thin Films and Nanotechnology, Thin Film Deposition Optics Electrical Engineering, Semiconductor Device, Physics Laser,

Nanoelectronics Electronic Engineering Biomedical Engineering, Sintering Microelectronics and Semiconductor Engineering, Microelectronics Detectors Absorption ,Conductivity of Silicon, Electrical Conductivity, XPS, Oxides Super conductors, Microelectronics Engineering, DTA, Semiconductor Engineering, Zinc Oxide, Photoelectron Spectroscopy Microelectromechanical Systems (MEMS) Vacuum Semiconductor Lase. 